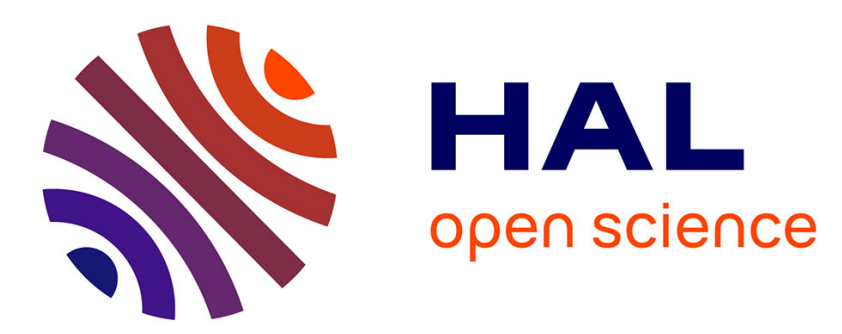

\title{
Peculiar clinicopathological features of immune-mediated necrotizing myopathies
}

Yves Allenbach, Olivier Benveniste

\section{To cite this version:}

Yves Allenbach, Olivier Benveniste. Peculiar clinicopathological features of immune-mediated necrotizing myopathies. Current Opinion in Rheumatology, 2018, 30 (6), pp.655-663. 10.1097/BOR.0000000000000547 . hal-03523499

\section{HAL Id: hal-03523499 \\ https://hal.sorbonne-universite.fr/hal-03523499}

Submitted on 12 Jan 2022

HAL is a multi-disciplinary open access archive for the deposit and dissemination of scientific research documents, whether they are published or not. The documents may come from teaching and research institutions in France or abroad, or from public or private research centers.
L'archive ouverte pluridisciplinaire HAL, est destinée au dépôt et à la diffusion de documents scientifiques de niveau recherche, publiés ou non, émanant des établissements d'enseignement et de recherche français ou étrangers, des laboratoires publics ou privés. 


\title{
Peculiar clinicopathological features of immune-mediated necrotizing myopathies
}

\author{
$Y_{v e s}$ Allenbach ${ }^{\mathrm{a}, \mathrm{b}}$ and Olivier Benveniste ${ }^{\mathrm{a}, \mathrm{b}}$
}

\begin{abstract}
Purpose of review
In the past decade, immune-mediated necrotizing myopathies have emerged as a separate entity in the heterogenous group of autoimmune myopathies. This group is characterized by clinical manifestations restricted to the muscle tissue, and until recently, the definition was based on muscular pathological features.
\end{abstract}

\begin{abstract}
Recent findings
It was shown that they are the most severe autoimmune myopathies in term of muscle damages. They have been associated with two myositis-specific antibodies: either anti-signal recognition particle (anti-SRP) or anti-hydroxy-3-methylglutaryl-CoA reductase (anti-HMGCR) antibodies. These two antibodies are now considered as immune-mediated necrotizing myopathy (IMNM) diagnostic criteria. Each antibody delineates a homogenous subgroup of IMNM patients in terms of severity and IMNM without myositisspecific antibodies have a high risk of malignancy. In addition, pathological observations as well as in-vitro experiments suggest the pathogenic role of anti-SRP and anti-HMGCR antibodies.
\end{abstract}

\begin{abstract}
Summary
IMNM are muscle-specific autoimmune diseases associated with a severe weakness and a risk poor muscle strength recovery. Anti-SRP and anti-HMGCR antibodies are specifically associated with this condition and are crucial for the diagnosis and the prognosis. The muscle biopsy remains necessary for IMNM diagnosis in absence of myositis-specific antibodies.
\end{abstract}

\section{Keywords}

autoantibodies, hydroxy-3-methylglutaryl-CoA reductase, myositis, necrotizing myopathies, signal recognition particle

\section{INTRODUCTION}

The spectrum of immune myopathies range from muscle-specific autoimmune diseases to systemic autoimmune diseases. Historically, they were classified into two categories, namely, polymyositis and dermatomyositis, based on the presence or absence of the characteristic dermatomyositis skin rash [1]. Nevertheless, both groups are heterogeneous, as patients may present with extramuscular manifestations and/or different types of myositis-specific autoantibodies [2].

Initially, in 1986, antisignal recognition particle (anti-SRP) antibody was identified in a subgroup of polymyositis patients [3]. Later, in 2002, it was shown that muscle biopsies from anti-SRP antibody positive (anti-SRP + ) patients showed the presence of necrotic muscle fibre without significant muscle inflammation [4]. One year later, a group of immune-mediated necrotizing myopathies (IMNMs) was recognized for the first time as a separate entity. The definition was based on pathological criteria showing predominant muscle fibre necrosis with no or mild muscle infiltrates [5]. On the basis of this definition, a new myositis-specific antibody targeting the hydroxy-3methylglutaryl-CoA reductase (HMGCR) protein was discovered in a subset of IMNM patients [6,7].

Now, IMNMs are a homogenous group of severe autoimmune muscle diseases without clinically relevant extramuscular manifestations. Yet, variations can be observed in IMNMs depending on serological status. This is one of the reasons why IMNM diagnosis criteria have been refined recently [ $\left.8^{*}\right]$ to distinguish anti-SRP+ IMNM, anti-HMGCR+ IMNM and antibody negative IMNM.

\footnotetext{
aSorbonne Universités, AP-HP, Hôpital Pitié Salpêtrière, Département de médecine Interne et Immunologie Clinique, Centre de Référence Maladies Neuro-Musculaires and ${ }^{b}$ INSERM - Centre de Recherche en Myologie - UMRS 974, Paris, France

Correspondence to Yves Allenbach, Département de médecine Interne et Immunologie Clinique, Centre de Référence Maladies Neuro-Musculaires, Hôpital Pitié Salpêtrière, 82 boulevard de l'hôpital, F-75013 Paris, France. Tel: +33 1421610 96; e-mail: Yves.allenbach@aphp.fr
}

Curr Opin Rheumatol 2018, 30:655-663

DOI:10.1097/BOR.0000000000000547 


\section{KEY POINTS}

- IMNM are the most severe autoimmune myopathies with a risk of poor muscle recovery.

- In presence of either anti-SRP or anti-HMGCR antibodies, the muscle biopsy is not necessary anymore to the diagnosis.

- For IMNM without myositis-specific antibodies, the muscle biopsy remains necessary for the diagnosis and this group has a high risk of malignancy.

- Pathological finding and in-vitro experiment suggest the pathological role of anti-SRP and anti-HMGCR antibody.

In this review, we aim to describe the clinicopathological features of INMN patients as well as prognosis, pathophysiology and therapeutic approaches, focusing on the recent advances in the field.

\section{MUSCLE CLINICAL PICTURE}

IMNMs represent $20-30 \%$ of patients suffering from auto-immune myositis $\left[9,10^{*}, 11\right]$, and the overall prevalence of myositis is 14 out of 10000 [12]. It can occur at any age, ranging from juvenile cases to elderly patients $[13,14]$. Most of the cases occur between 40 and 60 years of age, and women are more frequently affected $\left[7,9,10^{-}, 13\right]$.

Anti-HMGCR + IMNMs may be triggered by a statin exposure in one-half to two-thirds of the cases $[7,13]$. The statin exposure in IMNM patients varies depending on the age of onset; in patients older than 50 years, a statin exposure is observed in greater than $90 \%$ of the cases [7]. Interestingly, Dr. A. Mammen suggests that statins are also present in some foods and dietary supplements (oyster mushroom, red yeast rice or pu-erh tea), and this may trigger the disease, especially in Asian people in whom statin drug exposure in anti-IMNM patients is low [15].

At presentation, patients usually complain of muscle signs. Extramuscle manifestations are uncommon. There are two clinical phenotypes of the disease: patients with a subacute onset and patients with a slowly progressive disease.

Patients with a subacute onset are the most common and account for greater than two-thirds of the cases $[13,14]$. Typically, patients complain from a rapidly progressive muscle deficit occurring within weeks or months (less than 6 months) before the first hospital visit; they generally visit the Rheumatology department. In addition, myalgia is regularly reported (30-50\%) in IMNMs $\left[10^{*}, 13,16\right]$. One-quarter to one-third of anti-SRP+ or anti-HMGCR+ patients suffer from a slowly progressive onset of disease, greater than 6-12 months $[13,14]$. These later patients may present as dystrophic patients and are seen in Neurology/Myology departments.

Clinical examination shows a severe proximal, bilateral and symmetric muscle weakness. The hip flexor muscles are the most severely affected compared with other muscle groups in anti-SRP+ and anti-HMGCR+ patients $\left[10^{\boldsymbol{*}}, \mathbf{1 7}^{\boldsymbol{}}\right]$. Compared with other autoimmune myopathies, INMN patients more frequently harbour severe muscle weakness $\left[16,18^{-}\right]$. Nevertheless, there are some variations between anti-SRP+ and anti-HMGCR+ patients. Anti-SRP + patients suffer from a more severe muscle weakness than anti-HMGCR patients $\left[9,18^{\mathbf{*}}\right]$.

With a slowly progressive onset, muscle strength can be clinically considered normal (isolated increased creatine kinase level) for years, but more frequently, in addition to the muscle weakness, a muscle atrophy with some cases of winged scapula is observed [14], mimicking a limb girdle muscle dystrophy.

Life-threatening complications must always be considered. Swallowing troubles are frequent in IMNM patients, especially in anti-SRP+ patients where they occur in more than half of cases [9].

In addition, dyspnoea related to a weakness of the respiratory muscles may be observed. Usually, it occurs in very severely affected patients in whom exercise dyspnoea is difficult to demonstrate. The patients may complain of morning signs related to hypercapnia. This lung insufficiency can be screened for by nocturnal polygraphia, morning arterial gas measurements and pulmonary functional testing, ideally performed in both the sitting and supine position. Indeed, a more than 20\% decrease in vital capacity measured in the supine position versus the sitting position may be helpful in detecting severe or predominantly diaphragmatic weakness [19].

According to myopathological findings, the mean creatine kinase level in IMNM is higher than that observed in other myositis cases $\left[9,18^{-}, 20\right]$. Higher creatine kinase levels are also measured in anti-SRP + patients than anti-HMGCR + patients $\left[9,18^{\prime \prime}\right]$.

\section{DIAGNOSTIC TOOLS}

\section{Myositis antibody-detection}

In the case of autoimmune myositis, screening for myositis auto-antibodies is crucial, as by definition, 

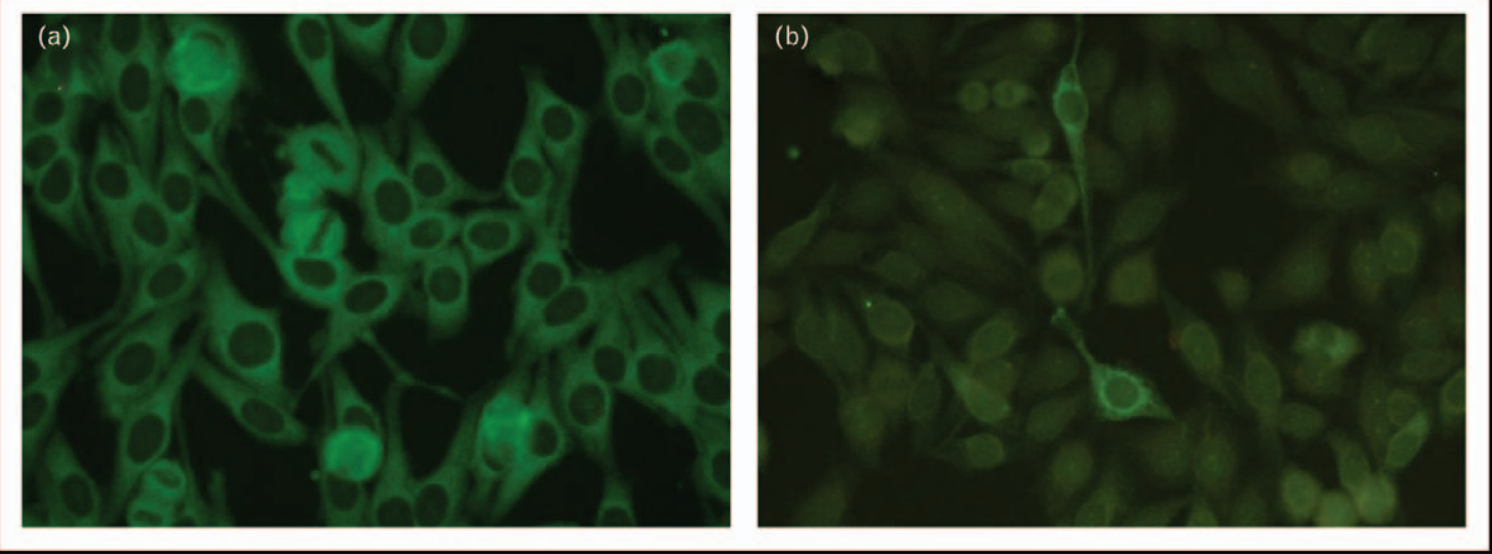

FIGURE 1. Anti-signal recognition particle and anti-hydroxy-3-methylglutaryl-CoA reductase auto-antibodies. (a) Indirect immunofluorescence anti-SRP antibody screening in serum from anti-SRP+ patients on HEp-2 cells shows diffuse positive cytoplasmic immunofluorescence on the majority of the cells. (b) Indirect immunofluorescence anti-HMGCR antibody screening in serum from anti-HMGCR + patients on HEp-2 cells shows a fine granular positive cytoplasmic immunofluorescence on the minority of the cells.

it is a key diagnostic tool and separates a homogeneous group of patients in terms of phenotype and prognosis [2]. In IMNM, anti-SRP and anti-HMGCR are considered (for the first time in autoimmune myopathies) a key diagnostic criterion [2]. In the case of IMNM, the clinician must test for anti-SRP as well as anti-HMGCR [21,22]. Screening for antinuclear antibodies was not sensitive enough to detect anti-SRP and anti-HMGCR antibodies using a screening test in HEp-2 cells [21,22]. SRP and HMGCR are intracytoplasmic proteins related to the endoplasmic reticulum, and indirect immunofluorescence may be negative (Fig. 1). SRP is key in the delivery of newly synthesized proteins [23], and HMGCR is a key enzyme in cholesterol biosynthesis [24]. There are different commercial kits for myositis-specific auto-antibody detection that have a limited risk of false-positive or false-negative results $[2,11,21,22]$. Immunoprecipitation remains the gold standard of auto-antibody detection, but it is not routinely feasible. For these reasons, the results of the tests must be challenged with the clinicopathological picture, and a retest can be performed using different methods if doubts exist.

\section{Muscle biopsy}

In the case of a typical clinicobiological picture of IMNMs with a positive detection of either anti-SRP or anti-HMGCR antibody, a muscle biopsy is not necessary to diagnose anti-SRP + or anti-HMGCR+ IMNM [ $\left.8^{*}\right]$. In other cases, a muscle biopsy must be performed.

The detection of the elementary lesions (muscle fibre, vascular, connective domains) and their distribution is important to recognize IMNMs (Fig. 2) [25]. Initially, IMNMs were defined on the basis of pathological criteria, including predominant muscle necrosis, whereas inflammatory infiltrates were mild or absent [5]. It has been shown that the percentage of necrotic muscle fibres is low in IMNMs (3.2\% in anti-SRP + patients vs. $1.8 \%$ in antiHMGCR patients, $P<0.05$ ) [18"]. The necrotic muscle fibres are randomly distributed, whereas they are confined in the perifascicular area in antisynthetase syndrome, another condition wherein significant muscle fibre necrosis is observed [18"]. However, indirect signs of necrosis, such as regenerating muscle fibres, are much more frequent [18"].

According to the first definition of IMNM, the inflammatory infiltrates are mild and mainly composed of macrophages $\left[9,18^{*}, 26\right]$. Nevertheless, significant inflammatory infiltrates with cell densities reaching the same range as those measured in other myositides are observed in one-quarter of IMNM cases (Fig. 2) [18"]. A subgroup of IMNM patients may have diffuse sarcolemmal major histocompatibility complex (MHC)-I positive staining. Together, those data showed that a subset of INMN patients have significant signs of muscle inflammation that are correlated with the percentage of necrotic muscle fibres [18"]. Thus, significant muscle inflammation is not considered an exclusion criterion in the new antibody-positive IMNM definition [8"].

The pathological criteria for auto-antibody negative IMNM are the presence of necrotic fibres and regenerative fibres (with different stages) with a scattered distribution and the presence of macrophage-predominant, paucilymphocytic infiltrates. Additional consistent features are sarcolemmal 
(a)

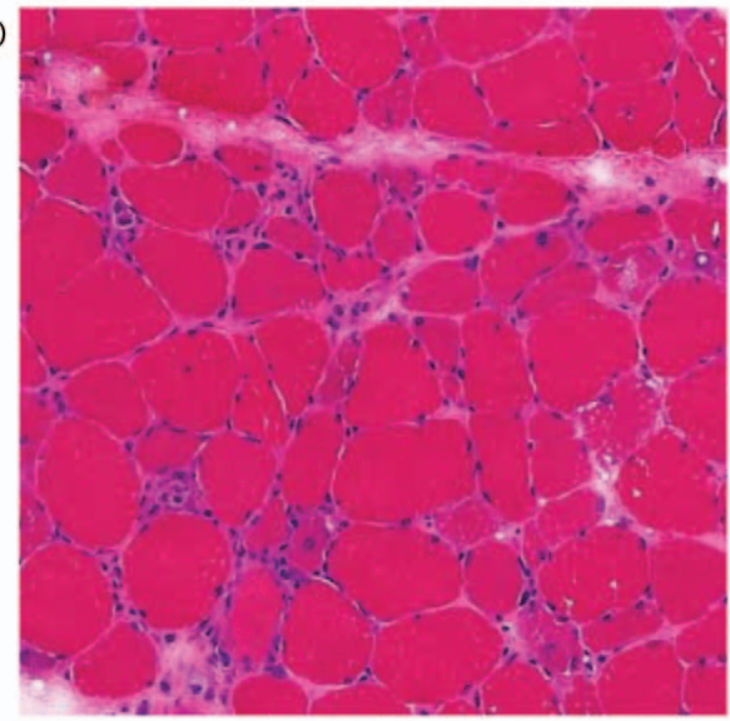

(c)

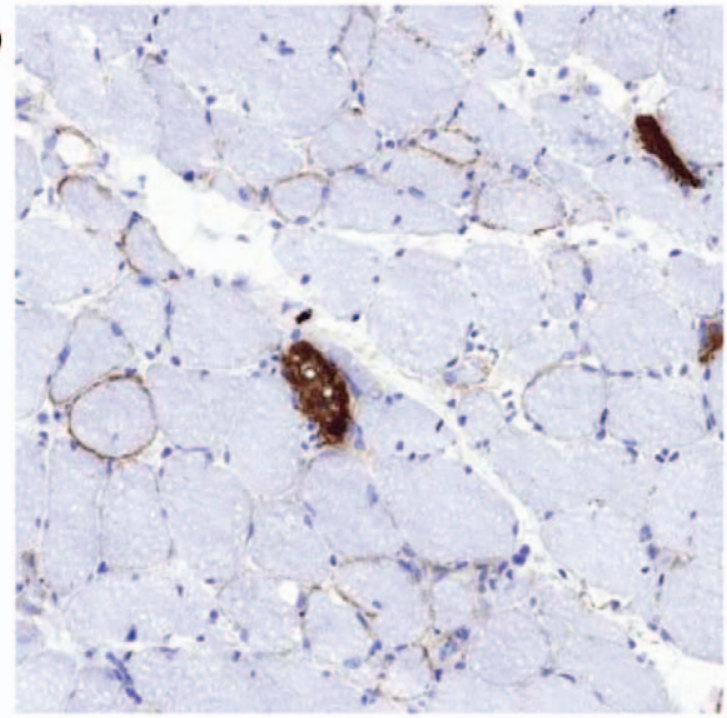

(b)

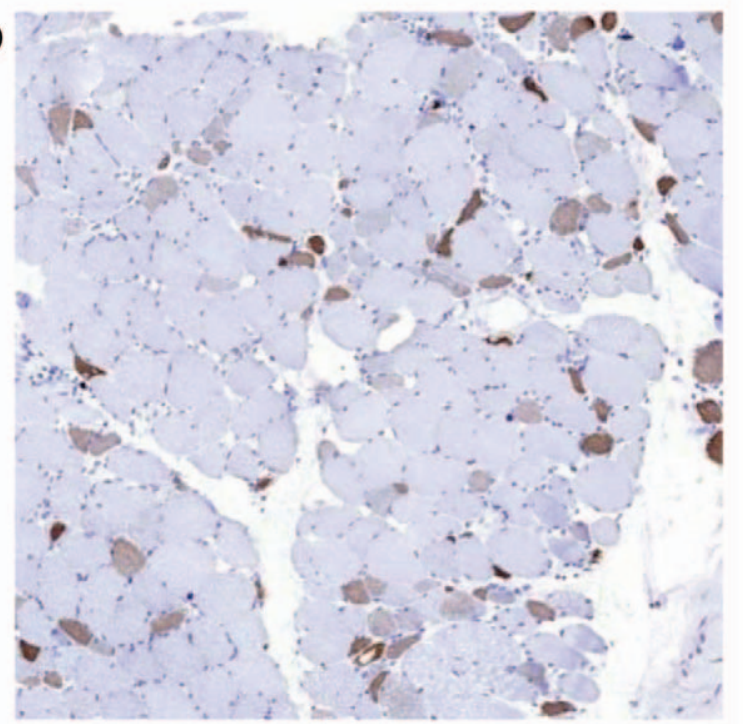

(d)

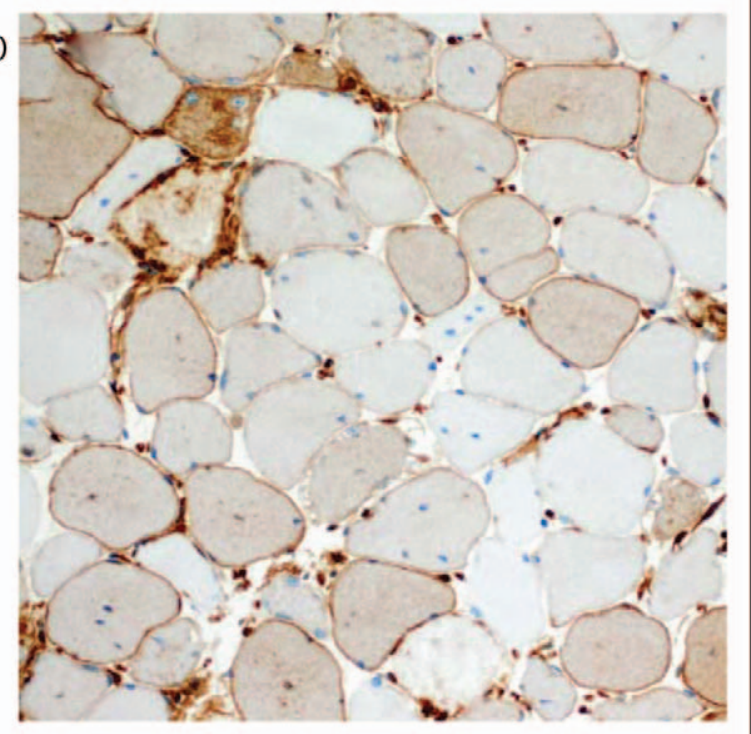

FIGURE 2. Pathological features of immune-mediated necrotizing myopathies. (a) Numerous necrotic muscle fibres randomly distributed in an IMNM muscle biopsy. Necrotic muscle fibres are at different stages of necrosis, ranging from pale hyalinized fibres to myophagocitosis. (HE staining). (b) High frequency of regenerating muscle fibres (positive neonate myosin heavy chain staining) randomly distributed. (c) Sarcolemmal C5b-9 deposits decorate nonnecrotic muscle fibres in INMM patients. Of note, nonspecific homogenous sarcoplasmic C5b-9 staining was found on necrotic muscle fibres. (d) Mild positive major histocompatibility complex I staining with a patchy distribution in IMNM patients on regenerating muscle fibres.

MHC class I expression (seen on nonnecrotic/ nonregenerating fibres), sarcolemmal complement deposition, endomysial fibrosis and proliferation, and enlarged capillaries [ $\left.8^{*}\right]$.

\section{EXTRASKELETAL MUSCLE FEATURES}

\section{Cardiac muscle features}

Conduction abnormalities, rhythm disorders and left ventricular dysfunction have been reported in $2-40 \%$ of anti-SRP cases $[14,16,20,27,28]$. If clear myocarditis has been reported in anti-SRP + patients, the significance and the specificity of all of the abovementioned cardiac manifestations remain to be established. Nevertheless, in our opinion, a systematic cardiac screening is necessary for anti-SRP patients. To date, cardiac involvement has not been clearly associated with anti-HMGCR patients.

\section{Extramuscular autoimmune features}

IMNM may be considered a pure muscle autoimmune disease. Specifically, in anti-HMGCR no auto-immune extra-muscle involvement has been 
reported. Anti-SRP+ patients do not suffer frequently from extramuscular manifestations, even though arthralgia (0-39\%) [14,20] and Raynaud's phenomenon $(0-26 \%)[20,29]$ have been reported. Anti-SRP IMNM may also result in an interstitial lung disease that is detected when a computed tomography (CT)-scan is performed (0-22\%) $[20,28]$. Again, this finding is not clinically significant and is not associated with pulmonary function test abnormalities.

On the contrary, it must be mentioned that some overlapping myositides may have myopathological features similar to IMNM, with diffuse randomly distributed necrotic/regenerating muscle fibres. This is the case in myositis-associated scleroderma [30].

\section{Malignancy in immune-mediated necrotizing myopathies}

An increased risk of malignancy in autoimmune myopathies is mainly observed in dermatomyositis, but it has also been reported in 'polymyositis' [31]. On the basis of this observation and previous case reports describing IMNM and cancer, the risk of malignancy in IMNM patients was compared with that expected in a sex and age-matched population. It was shown that patients with antibody-negative IMNM have a higher risk of malignancy, whereas the risk was similar for anti-SRP patients [32"]. A mild increase was observed in two studies of antiHMGCR+ patients [32",33], but this was not observed by others [17"]. In the case of cancer association, malignancy occurs within 3 years before or after the diagnosis and in most cases within 1 year of diagnosis [32"]. Cancer affects mainly patients older than 50 years. No specific type of cancer is observed in IMNM, and patients with malignancy have a lower survival rate [32"].

\section{IMMUNE-MEDIATED NECROTIZING MYOPATHY PROGNOSIS}

Patients suffering from autoimmune myopathies have a higher mortality rate than the general population [34]. The main causes of death in autoimmune myopathies are malignancy and diseases of the respiratory and circulatory systems [34]. All of these life-threatening complications, except severe interstitial lung disease, may occur in IMNM patients.

One-quarter of IMNM patients suffer from difficulties in their daily living, graded as modified Rankin Scale scores of 3-5 [9]. Only 50\% of anti$\mathrm{SRP}+$ patients reached near-full or full strength after 4 years of treatment, and a younger age at onset is associated with more severe weakness [10"]. Only
$44 \%$ of anti-HMGCR + patients reached full strength with immunosuppressive therapy, and younger patients had more severe disease and a worse prognosis than older patients [17"]. Of note, statin exposure was not independently associated with the rate of muscle strength improvement [17"].

These observations are in line with the presence of significant muscle damage in IMNM. Compared with the other myositides, IMNM was characterized by a higher proportion of thigh muscles with oedema, atrophy and fatty replacement (Fig. 3) [35"']. According to the clinicobiological observations, among IMNM, anti-SRP+ patients had more atrophy and fatty replacement than anti-HMGCR+ patients [35"'].

Together, the data show that IMNMs are the most severe group of myositides with a poor outcome in terms of muscle strength compared with dermatomyositis or the antisynthetase syndrome.

\section{PATHOPHYSIOLOGY}

The pathophysiology of IMNM is not fully understood. Some human leukocyte antigen (HLA) class I and II antigens have been associated with IMNM [36-38]. Statin exposure is another factor associated with anti-HMGCR+ IMNM onset, suggesting that in genetically predisposed patients, the drug may trigger the disease. It has been shown that HMGCR expression is upregulated in regenerating myofibers in anti-HMGCR IMNM patients [7]. Similarly, HMGCR and SRP proteins were detected at the sarcolemmal level in IMNM patients [18"]. This observation and the presence of membrane attack complexes at the surface of nonnecrotic muscle fibres suggest a pathogenic role of the auto-antibodies. This hypothesis is reinforced by the observation of IgG and C1q at the sarcolemmal level in IMNM patients, suggesting classical pathway activation (antibody-dependant) of complement [18"]. The titre of the auto-antibodies correlates with the disease activity of anti-SRP+ and anti-HMGCR+ myositis $\left[13,17^{*}, 22\right]$. In addition, it has been shown in vitro that anti-SRP and anti-HMGCR induce muscle fibre atrophy and impair muscle regeneration [39"']. Together, these observations suggest a pathogenic role of auto-antibodies in IMNM.

\section{TREATMENT}

IMNM treatment remains challenging. There has been no randomized clinical trial specifically designed for IMNM patients. Retrospective studies have shown that anti-SRP and anti-HMGCR patients have a long disease duration with frequent relapses when using several treatments, including 


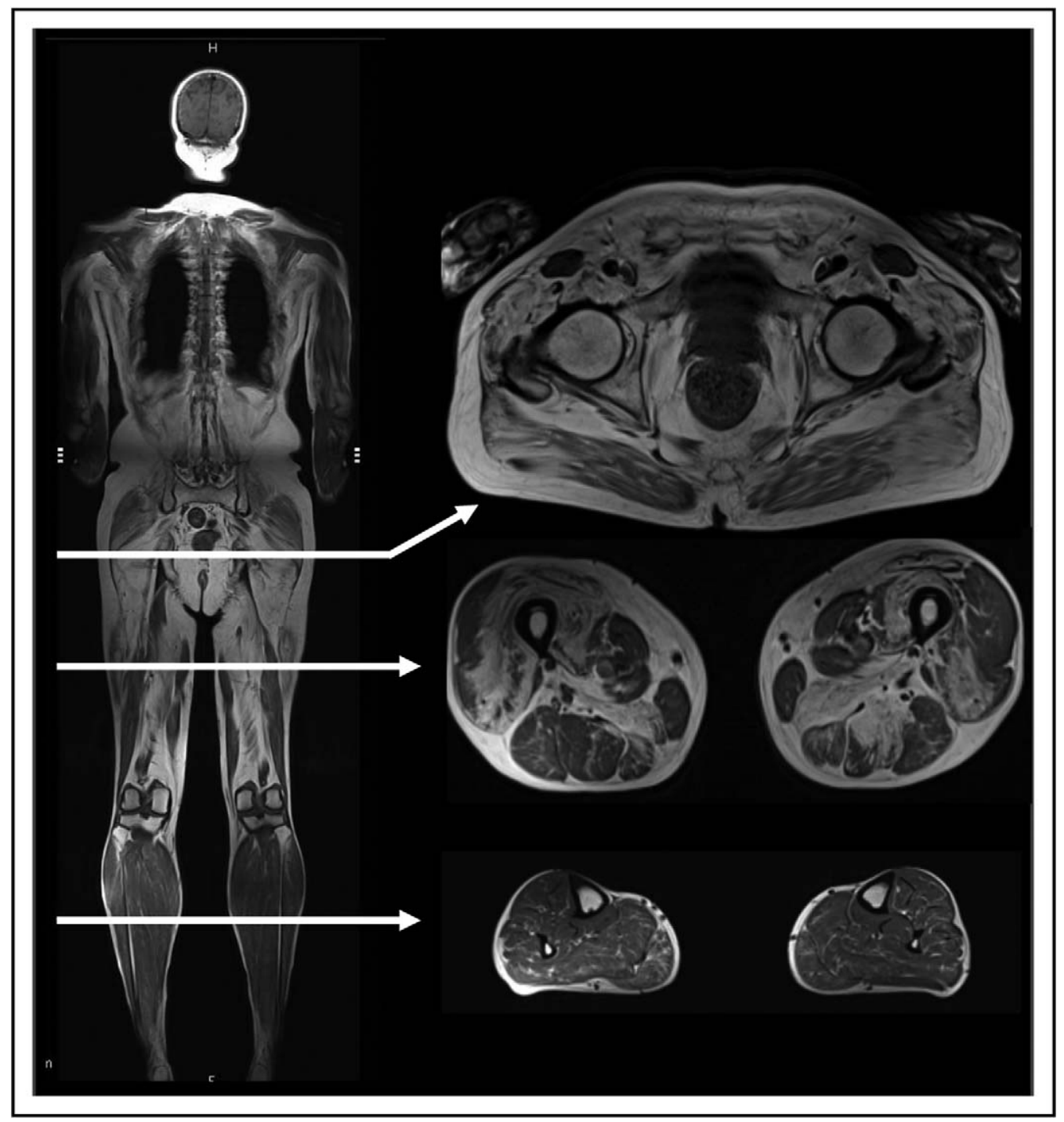

FIGURE 3. Immune-mediated necrotizing myopathy whole-body MRI. Whole-body MRI (T1) of a severely affected IMNM patients show important muscle damages in the lower limbs. The important fatty replacement (T1 hypersignal) is observed in the gluteus muscles as well as in the thigh muscles, whereas the muscles of the lowers limbs are spared.

corticosteroids and immunosuppressants [9,10", $\left.13,1^{-}\right]$. It was shown that rituximab administration was efficacious in $75 \%$ of cases $\left[10^{-}, 40\right]$, leading to the consideration of its use as a first-line treatment in anti-SRP patients [8"]. In anti-HMGCR+ patients, intravenous immunoglobulin has been efficacious as a monotherapy [41], showing its importance in the treatment strategy for those patients [8"].

The first-line treatment strategy must be adapted to each patients' condition and the severity of the muscle disease. The recommendations of the 224th ENMC are shown in Fig. 4.

Disease assessment is challenging in autoimmune myositis. The ACR/EULAR recommendations have been updated to capture a significant improvement in the domains (e.g. muscle, skin, lung or joints) that could be affected in myositis patients [42]. In anti-SRP + and anti-HMGCR+ IMNM, it was shown that the creatine kinase level is associated with disease activity $\left[13,17^{\prime}\right]$, as the creatine kinase level mirrors the percentage of necrotic muscle fibres [18"]. Regarding the severe muscle damage observed in IMNM patients [35"'], it seems logical to target normal creatine kinase levels to treat IMNM and determine disease remission.

In addition, a slight increase in creatine kinase levels may be a sign of important and persistent disease activity in severely affected patients, as a normal creatine kinase level is correlated with muscle mass. Indeed, IMNM patients with important clinically defined sarcopenia and low creatinine blood levels may have subnormal creatine kinase levels, whereas persistent disease activity is determined by muscle oedema using MRI. 


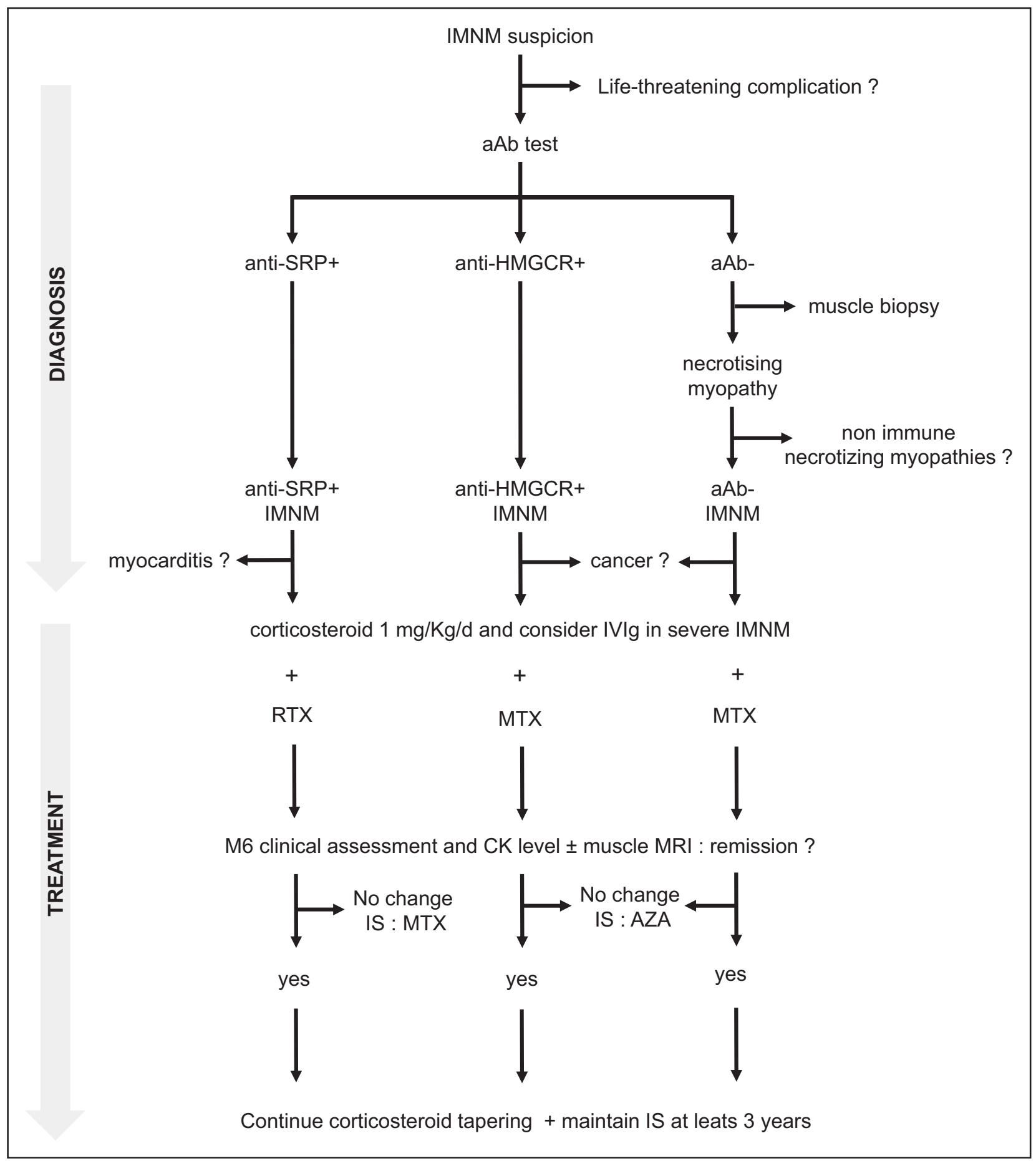

FIGURE 4. Immune-mediated necrotizing myopathy diagnostic and treatment strategy. The life-threatening complications of IMNMs are swallowing trouble and/or dyspnoea. The causes of nonimmune necrotizing myopathies are acquired necrotizing myopathies (drug/toxic, infections and endocrine/metabolic) and genetic myopathies (muscular dystrophy in case of slowly progressive onset and metabolic myopathies in case of acute onset). Severe IMNM cases are patients with dysphagia and/or walking difficulty. aAb, auto-antibodies; AZA, azathioprine; IS, immunosuppressant; MTX, methotrexate; RTX, rituximab.

MRI of the muscle is an important tool to monitor IMNM patients, as MRI permits the measurement of disease activity and also muscle damage. MRI is sometimes used to avoid treatment escalation in patients with definitive fixed muscle damage with fatty replacement.

To conclude, IMNM is the most severe autoimmune muscular disease with regard to patient 
impairment and the risk of muscle damage. IMNM can be anti-SRP+, anti-HMGCR+ or auto-antibody negative. This serological distinction allows the determination of a more definitive phenotype in the patient (more severe in anti-SRP patients), the determination of potential cancer association (mainly in antibody-negative IMNM) and the development of treatment strategies.

\section{Acknowledgements}

We want to acknowledge Drs. Jean-Luc Charuel and Lucile Musset for having provided the pictures of immunofluorescence of myositis-specific antibodies. Similarly, we thank Professor Pierre Carlier and Jean-Marc Boisserie for the MRI pictures of IMNM patients they gave to illustrate the muscle damages.

\section{Financial support and sponsorship}

None.

\section{Conflicts of interest}

There are no conflicts of interest.

\section{REFERENCES AND RECOMIMENDED \\ READING}

Papers of particular interest, published within the annual period of review, have been highlighted as:

- of special interest

a of outstanding interest

1. Bohan A, Peter JB. Polymyositis and dermatomyositis (first of two parts). $N$ Engl J Med 1975; 292:344-347.

2. Benveniste $O$, Stenzel $W$, Allenbach $Y$. Advances in serological diagnostics of inflammatory myopathies. Curr Opin Neurol 2016; 29:662-673.

3. Reeves $W H$, Nigam SK, Blobel G. Human autoantibodies reactive with the signal-recognition particle. Proc Natl Acad Sci U S A 1986; 83:9507-9511.

4. Miller T, Al-Lozi M, Lopate G, Pestronk A. Myopathy with antibodies to the signal recognition particle: clinical and pathological features. J Neurol Neurosurg Psychiatry 2002; 73:420-428.

5. Hoogendijk JE, Amato AA, Lecky BR, et al. 119th ENMC international workshop: trial design in adult idiopathic inflammatory myopathies, with the exception of inclusion body myositis, 10-12 October 2003, Naarden, The Netherlands. Neuromuscul Disord NMD 2004; 14:337-345.

6. Christopher-Stine L, Casciola-Rosen LA, Hong G, et al. A novel autoantibody recognizing $200-k d$ and $100-k d$ proteins is associated with an immunemediated necrotizing myopathy. Arthritis Rheum 2010; 62:2757-2766.

7. Mammen AL, Chung T, Christopher-Stine L, et al. Autoantibodies against 3 hydroxy-3-methylglutaryl-coenzyme a reductase (HMGCR) in patients with statin-associated autoimmune myopathy. Arthritis Rheum 2011; 63:713-721.

8. Allenbach $\mathrm{Y}$, Mammen $\mathrm{AL}$, Stenzel $\mathrm{W}$, Benveniste O. Immune-mediated

- necrotizing Myopathies Working Group. 224th ENMC International Workshop: Clinico-sero-pathological classification of immune-mediated necrotizing myopathies Zandvoort, The Netherlands, 14-16 October 2016. Neuromuscul Disord 2018; 28:87-99.

In this conference, an international consensus was reached to refine IMNM criteria.

For the first time, IMNM definition is based on serological criteria.

9. Watanabe $Y$, Uruha A, Suzuki S, et al. Clinical features and prognosis in antiSRP and anti-HMGCR necrotising myopathy. J Neurol Neurosurg Psychiatry 2016; 87:1038-1044.

10. Pinal-Fernandez I, Parks $C$, Werner JL, et al. Longitudinal course of disease in

- a large cohort of myositis patients with autoantibodies recognizing the signal recognition particle. Arthritis Care Res 2017; 69:263-270.

In this article, the authors described for the first time the precise muscle phenotype

of anti-SRP+ patients. For the first time, they have clearly demonstrated the poor muscle outcome.

11. Mammen AL, Pak K, Williams EK, et al. Rarity of anti3-hydroxy-3-methylglutaryl-coenzyme A reductase antibodies in statin users, including those with self-limited musculoskeletal side effects. Arthritis Care Res Hoboken 2012; 64:269-272.

12. Meyer $A$, Meyer $N$, Schaeffer $M$, et al. Incidence and prevalence of inflammatory myopathies: a systematic review. Rheumatol Oxf Engl 2015; 54:50-63.
13. Allenbach $Y$, Drouot $L$, Rigolet $A$, et al. Anti-HMGCR autoantibodies in European patients with autoimmune necrotizing myopathies: inconstant exposure to statin. Medicine (Baltimore) 2014; 93:150-157.

14. Suzuki S, Hayashi YK, Kuwana M, et al. Myopathy associated with antibodies to signal recognition particle: disease progression and neurological outcome. Arch Neurol 2012; 69:728-732.

15. Pinal-Fernandez I, Casal-Dominguez M, Mammen AL. Immune-mediated necrotizing myopathy. Curr Rheumatol Rep 2018; 20:21.

16. Kao $\mathrm{AH}$, Lacomis $\mathrm{D}$, Lucas $\mathrm{M}$, et al. Antisignal recognition particle autoantibody in patients with and patients without idiopathic inflammatory myopathy. Arthritis Rheum 2004; 50:209-215.

17. Tiniakou E, Pinal-Fernandez I, Lloyd TE, et al. More severe disease and slower

- recovery in younger patients with anti3-hydroxy-3-methylglutaryl-coenzyme $A$ reductase-associated autoimmune myopathy. Rheumatol Oxf Engl 2017; 56:787-794.

In this article, the authors described for the first time the precise muscle phenotype of anti-HMGCR + patients. For the first time, they have clearly demonstrated the poor muscle outcome, stratifying the outcome based on the age of onset.

18. Allenbach $Y$, Arouche-Delaperche $L$, Preusse $C$, et al. Necrosis in anti-SRP+

- and anti-HMGCR+myopathies: role of autoantibodies and complement. Neurology 2018; 90:e507-e517.

In this article, the authors described myopathological changes IMNM with a quantitative approach. This study shows IgG and complement deposits at the sarcolemmal level and suggest a pathogenic role of auto-antibody in muscle fibre necrosis.

19. Fromageot $C$, Lofaso $F$, Annane $D$, et al. Supine fall in lung volumes in the assessment of diaphragmatic weakness in neuromuscular disorders. Arch Phys Med Rehabil 2001; 82:123-128.

20. Hengstman GJD, ter Laak HJ, Egberts WTMV, et al. Antisignal recognition particle autoantibodies: marker of a necrotising myopathy. Ann Rheum Dis $2006 ; 65: 1635-1638$

21. Drouot $L$, Allenbach $Y$, Jouen $F$, et al. Exploring necrotizing autoimmune myopathies with a novel immunoassay for anti3-hydroxy-3-methyl-glutarylCoA reductase autoantibodies. Arthritis Res Ther 2014; 16:R39.

22. Benveniste $O$, Drouot $L$, Jouen $F$, et al. Correlation of antisignal recognition particle autoantibody levels with creatine kinase activity in patients with necrotizing myopathy. Arthritis Rheum 2011; 63:1961-1971.

23. Saraogi I, Shan S. Molecular mechanism of co-translational protein targeting by the signal recognition particle. Traffic Cph Den 2011; 12:535-542.

24. Goldstein JL, DeBose-Boyd RA, Brown MS. Protein sensors for membrane sterols. Cell 2006; 124:35-46.

25. Allenbach $Y$, Benveniste $\mathrm{O}$, Goebel $\mathrm{H}-\mathrm{H}$, Stenzel W. Integrated classification of inflammatory myopathies. Neuropathol Appl Neurobiol 2017; 43:62-81.

26. Chung T, Christopher-Stine L, Paik JJ, et al. The composition of cellular infiltrates in anti-HMG-CoA reductase-associated myopathy. Muscle Nerve 2015; 52:189-195.

27. Targoff IN, Johnson AE, Miller FW. Antibody to signal recognition particle in polymyositis. Arthritis Rheum 1990; 33:1361-1370.

28. Basnayake SK, Blumbergs $P$, Tan JA, et al. Inflammatory myopathy with antiSRP antibodies: case series of a South Australian cohort. Clin Rheumatol $2015 ; 34: 603-608$.

29. Wang L, Liu L, Hao $\mathrm{H}$, et al. Myopathy with antisignal recognition particle antibodies: clinical and histopathological features in Chinese patients. Neuromuscul Disord 2014; 24:335-341.

30. Ranque B, Authier FJ, Berezne A, et al. Systemic sclerosis-associated myopathy. Ann N Acad Sci 2007; 1108:268-282.

31. Zahr ZA, Baer AN. Malignancy in myositis. Curr Rheumatol Rep 2011; 13:208-215.

32. Allenbach $Y$, Keraen J, Bouvier A-M, et al. High risk of cancer in autoimmune

- necrotizing myopathies: usefulness of myositis specific antibody. Brain J Neurol 2016; 139:2131-2135.

In this study, the authors have compared the prevalence of malignancy in the three IMNM subtypes (anti-SRP+, anti-HMGCR+ and seronegative) to the aged and sex-marched population. They have showed an important increased risk in antibody-negative IMNM, whereas the risk is mild in anti HMGCR+ patients.

33. Kadoya $\mathrm{M}$, Hida $\mathrm{A}$, Hashimoto Maeda $\mathrm{M}$, et al. Cancer association as a risk factor for anti-HMGCR antibody-positive myopathy. Neurol Neuroimmunol Neuroinflammation 2016; 3:e290.

34. Dobloug $\mathrm{C}$, Garen $\mathrm{T}$, Bitter $\mathrm{H}$, et al. Prevalence and clinical characteristics of adult polymyositis and dermatomyositis; data from a large and unselected Norwegian cohort. Ann Rheum Dis 2015; 74:1551-1556.

35. Pinal-Fernandez I, Casal-Dominguez M, Carrino JA, et al. Thigh muscle MRI in

- immune-mediated necrotising myopathy: extensive oedema, early muscle damage and role of anti-SRP autoantibodies as a marker of severity. Ann Rheum Dis 2017; 76:681-687.

This study has reported for the first time the muscle damages occurring in a large cohort of IMNM compared with others myositis. According to the poor outcomes of IMNM, the authors showed the important fatty replacement in IMNM patients compared with the other group of IMNM.

36. Mammen AL, Gaudet D, Brisson $D$, et al. Increased frequency of $\mathrm{DRB} 1 * 11: 01$ in anti-HMG-CoA reductase-associated autoimmune myopathy. Arthritis Care Res (Hoboken) 2012; 64:1233-1237.

37. Kishi T, Rider LG, Pak K, et al. Association of anti3-hydroxy-3-methylglutarylcoenzyme A reductase autoantibodies with DRB1 $* 07: 01$ and severe myositis in juvenile myositis patients. Arthritis Care Res 2017; 69: 1088-1094. 
38. Ohnuki Y, Suzuki S, Shiina T, et al. HLA-DRB1 alleles in immune-mediated necrotizing myopathy. Neurology 2016; 87:1954-1955.

39. Arouche-Delaperche L, Allenbach $Y$, Amelin D, et al. Pathogenic role of

- antisignal recognition protein and anti3-Hydroxy-3-methylglutaryl-CoA reductase antibodies in necrotizing myopathies: myofiber atrophy and impairment of muscle regeneration in necrotizing autoimmune myopathies. Ann Neurol 2017; 81:538-548.

In this study, the authors test in vitro the pathogenic role of the anti-SRP and antiHMGCR antibodies. For the first time, the authors showed that they can induce muscle atrophy and impair muscle regeneration.
40. Valiyil R, Casciola-Rosen L, Hong G, et al. Rituximab therapy for myopathy associated with antisignal recognition particle antibodies: a case series. Arthritis Care Res Hoboken 2010; 62:1328-1334.

41. Mammen AL, Tiniakou E. Intravenous immune globulin for statintriggered autoimmune myopathy. N Engl J Med 2015; 373:16801682.

42. Rider LG, Ruperto N, Pistorio A, et al. 2016 ACR-EULAR adult dermatomyositis and polymyositis and juvenile dermatomyositis response criteria: methodological aspects. Rheumatol Oxf Engl 2017; 56:18841893. 